\title{
Wirtschaftsförderung bei rückläufigen Finanzbudgets - Überlegungen zur leistungsorientierten Maßnahmenanpassung
}

\author{
Klaus-Peter Schulze
}

\section{Die Handlungsspielräume der öffentlichen Wirtschaftsförderung werden enger}

Wir alle wissen: Der Staat wird immer ärmer. Steuermindereinnahmen, steigende Zinslast, zunehmende Kosten drücken die Verantwortlichen im öffentlichen Bereich und schränken ihren Handlungsspielraum zunehmend ein. Öffentliche Haushaltsdebatten werden zum Verteilungskampf um einen immer kleiner werdenden Kuchen. Heute heißt es mehr denn je, Prioritäten zu setzen, staatliche Leistungen auf die notwendigen Kernaufgaben zu reduzieren.

Dies gilt auch für den Bereich der Wirtschaftsförderung als ein Aufgabenbereich staatlichen Handelns. Immer wieder werden Fälle unangemessenen Mitteleinsatzes fuir einzelne Wirtschaftsförderaufgaben publik. Die Hoffnung auf den vermeintlich „großen Wurf“ oder einfach das uniberlegte „weiter wie bisher" sind risikoreich und können vor allem Chancen in anderen mehr Erfolg versprechenden Bereichen verbauen. Wirtschaftsförderung heute kann sich keine finanziellen Abenteuer mehr leisten. Mehr denn je geht es darum, die knappen öffentlichen Kapazitäten so aufzuteilen, dass sie die größten Erfolge für die künftige Wirtschaftsentwicklung erwarten lassen. Drei miteinander verknüpfte Aktionsfelder sind dabei auszumachen:

- Prioritätensetzung durch inhaltliche Konzentration auf zukunftsträchtige Wirtschaftsförderbereiche,

- Anpassung des Instrumenteneinsatzes bei rückläufigen Finanzbudgets,

- Erhöhung der Selbstfinanzierungskraft im Wirtschaftsförderbereich.

Hinsichtlich der inhaltlichen Profilierung wird auf die vom Verfasser an anderer Stelle vorgestellten Überlegungen verwiesen [1]. Beiträge zur Bereinigung des Instrumenteneinsatzes werden in den nachfolgenden Erörterungen entwickelt. Maßnahmen zur Erhöhung der Selbstfinanzierungskraft müssen späteren Ausfuihrungen überlassen bleiben.

Grundsätzlich gilt: Bei abnehmenden Haushaltsvolumina sind die knappen Wirtschaftsfördermittel auf die Instrumente zu konzentrieren, die den größten Wirtschaftsfördererfolg erwarten lassen. Hierzu bedarf es abwägender Kosten-Nutzen-Überlegungen in Bezug auf die einzusetzenden Wirtschaftsförderinstrumente.

\section{Die Instrumente staatlicher Wirtschafts- förderung und deren Erfolgsmaßstäbe}

Zukunftsorientierte Wirtschaftsförderung leistet einen Beitrag, die Wettbewerbsfähigkeit der regionalen Wirtschaft zu erhöhen. Aufgrund der strategischen Bedeutung der Innovationskraft für die regionale Wirtschaftsentwicklung gilt ein innovatives Wirtschaftsklima als zentraler Standortfaktor. Zu seiner Entwicklung greifen Ansiedlungsbemühungen, Unternehmensentwicklung und Existenzgründungsunterstuitzung ineinander. Alle drei Wirtschaftsförderfelder bilden zusammen den integrierten Ansatz einer ganzheitlichen Wirtschaftsförderung.

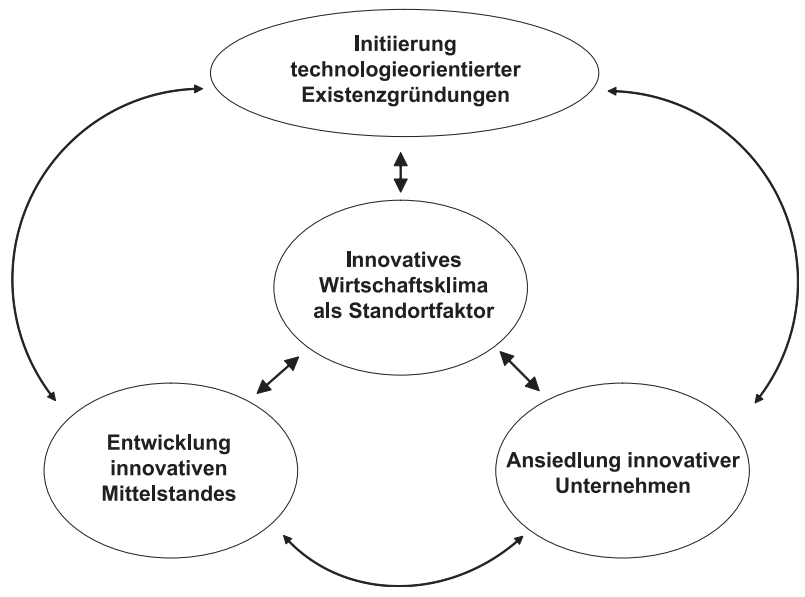

Abb. 1: Regelkreis zukunftsorientierter Wirtschaftsförderung

Jedem Wirtschaftsförderfeld kann eine spezifische Gruppe von wirtschaftsfördernden Instrumentenbündeln zugeordnet werden. Jedes Instrumentenbündel lässt sich in eine Vielzahl weiterer Handlungsmaßnahmen aufgliedern, die sich problem- und zielgruppenspezifisch unterscheiden. Organisatorisch sind die einzelnen Instrumentenbündel in aller Regel eigenständigen Rechtsformen zugeordnet. Nur in zwei deutschen Bundesländern sind sie zurzeit organisatorisch zusammengefasst: in Bremen und in Brandenburg.

Unabhängig von der Organisationsform ist es aus der übergeordneten Sicht staatlicher Wirtschaftsförderung notwendig, den einzelnen Instrumentenbündeln und damit ihren Trägern Finanzausstattungen zuzuordnen. Den Eingangsüberlegungen folgend spielt bei der Bemessung der Finanzausstattung der aus dem Instrumenteneinsatz erwartete Nutzen die entscheidende Rolle. Während der Input staatlicher Wirtschaftsförderung als Ausgaben bzw. 
Kosten eindeutig quantifizierbar ist, gibt es zur Messung des Outputs unterschiedliche Ansätze.

Im Wirtschaftsförderfeld „Ansiedlung“ ist als Erfolgsmaßstab die Anzahl der durch die Maßnahmen neu geschaffenen bzw. erhaltenen Arbeitsplätze gebräuchlich. Auch im Bereich der Existenzgründung findet dieser Maßstab gelegentlich Anwendung. Im Bereich der innovativen Unternehmensentwicklung findet man häufig Kriterien wie Patentanmeldung, FuE-Aufwendungen bezogen auf den Umsatz oder FuE-Personal bezogen auf das Gesamtpersonal.

Der Vorteil der Arbeitsplatzzählung als Erfolgsindikator liegt sicher in seiner einfachen Verständlichkeit, der politischen Vermittelbarkeit und der quantifizierbaren Verwendbarkeit. Bei näherem Hinsehen werden die Schwächen dieses Outputindikators jedoch rasch deutlich. Undifferenzierte Arbeitsplatzzählungen

- klammern Strukturentwicklungseffekte aus,

- beruicksichtigen keine einzelwirtschaftlichen Wertschöpfungsprozesse,

- können als Zielgrößen Wirtschaftsförderprozesse in die falsche Richtung steuern.

Eine undifferenzierte Arbeitsplatzzählung suggeriert: Arbeitsplatz ist gleich Arbeitsplatz. Mit dieser einfachen Logik werden neu geschaffene High-Tech-Arbeitsplätze mit einfachen Dienstleistungsarbeitsplätzen gleichgesetzt. Angesichts des weltweiten Strukturwandels zur Wissensgesellschaft ist diese Vorgehensweise ein höchst fragwürdiges Unterfangen.

Arbeitsplätze sind einzelwirtschaftliche Kostenfaktoren. Kosten reduzieren die Wertschöpfung - vor allem in Ländern relativ hoher Arbeitskosten wie Deutschland. Eine geringe Wertschöpfung macht Unternehmen konjunkturanfällig und beeinträchtigt ihre internationale Konkurrenzfähigkeit. Bevorzugt die Wirtschaftsförderung arbeitsplatzintensive Unternehmen, besteht die Gefahr, dass sie Strukturen stuitzt, deren Konkurrenzfähigkeit auf Dauer nicht gegeben ist.

Arbeitsplatzschaffung als alleinige Zielgröße wirtschaftsfördernder Aktivitäten beinhaltet damit die Gefahr der Fehlallokation der Ressourcen. Auf der Jagd nach hohen Arbeitsplatzzahlen werden notwendige Umstrukturierungsprozesse nicht angegangen, die Unterstuitzung einzelbetrieblicher Wertschöpfungsprozesse wird vernachlässigt, die Aufmerksamkeit wird von nicht zukunftsbezogenen Wirtschaftsbereichen abgelenkt.

Auch für das Stabilitäts- und Wachstumsgesetz (StWG), das wirtschaftsförderndes Handeln bundesweit legitimiert, ist die Beschäftigungsförderung nur eines von drei Zielkriterien.

Angesichts dieser Probleme werden zunehmend Stimmen laut, die auf eine Umorientierung bei der Erfolgsmessung im Wirtschaftsförderbereich drängen. Vorgeschlagen werden u. a. mehrdimensionale Kennzahlensysteme, die in die Erfassung der Brutto- wertschöpfung je Einwohner münden, oder Kriterien, die einzelwirtschaftliche Umsatzsteigerungen berücksichtigen bzw. nominal erzielte strukturwirtschaftliche Effekte in den Regionen einbeziehen [2]. Eine auf Überwindung struktureller Nachteile gerichtete Wirtschaftsförderung kann daher auf die Einbeziehung struktureller Effekte bei der Bewertung des Wirtschaftsfördererfolges nicht verzichten.

\section{Die Berücksichtigung struktureller Effekte bei der Ermittlung des Wirtschaftsfördererfolges}

Bei der Ermittlung der internationalen Konkurrenzfähigkeit einer Volkswirtschaft spielt deren innovatives Potenzial eine immer wichtigere Rolle. In jährlich wiederkehrenden Analysen legt das Bundesministerium für Bildung und Forschung seinen Bericht zur Technologischen Leistungsfähigkeit der Bundesrepublik Deutschland vor [3]. Grundlage der Analyse ist die Strukturierung der Wirtschaftsbereiche in die Kategorien „Spitzentechnologie“, „Hochwertige Technologie“ und „Wissensintensive Dienstleistung“ [4]. Ein struktureller Vergleich mit den Volkswirtschaften anderer Staaten macht so Stärken und Schwächen der einheimischen Wirtschaft deutlich. Die aktuellen Zahlen für Deutschland zeigen insgesamt gegenüber den Vorjahren in vielen Bereichen eine rückläufige Tendenz.

Die in der genannten Weise für Gesamtdeutschland zugrunde gelegte Metrik des Strukturvergleiches lässt sich auch auf Bundesländerebene herunterbrechen und als Grundlage für die Messung des regionenbezogenen Wirtschaftsfördererfolges verwenden. Durch Gewichtung der Erfolgsindikatoren (z. B. geschaffene und erhaltene Arbeitsplätze) nach Maßgabe ihres Innovationsgehaltes lassen sich Struktureffekte abbilden. So wird etwa die Schaffung eines einfachen Arbeitsplatzes in einem Call-Center geringer gewichtet als ein hoch spezialisierter Arbeitsplatz in der Branche Luftfahrttechnik. Bei der Zuordnung kann auf die einschlägige Einteilung des Bundesministeriums für Bildung und Forschung zurüickgegriffen werden, die die jeweilige Branche der amtlichen Wirtschaftsplansystematik nach ihrem jeweiligen Technologie- und Wissensgehalt gliedert [5].

Einer besonderen Sorgfalt bedarf die Bestimmung der Gewichtungsfaktoren. Anhaltspunkte fuir deren Bemessung liefern u. a. die fiskalischen Effekte geschaffener Arbeitsplätze. Nach Berechnungen der Prognos AG bringt ein durchschnittlicher neuer Arbeitsplatz im Bundesland Berlin ca. 3.900 Euro an Steuermehreinnahmen, ein hoch qualifizierter, uiberdurchschnittlicher Arbeitsplatz hingegen über 4.800 Euro - jeweils vor Länderfinanzausgleich [6]. Damit wäre eine um ca. 23 \% höhere Gewichtung von Hightech-Arbeitsplätzen ableitbar. Weitere Gesichtspunkte, wie technologiestrukturelle Entwicklungsprognosen oder wirtschaftspolitische Schwerpunktsetzungen, können in die letztendliche Gewichtungsentscheidung einbezogen werden. 
Im Ergebnis ermöglicht der aufgezeigte Weg die Einbeziehung struktureller Effekte bei der Ermittlung des Wirtschaftsfördererfolges. Damit können wesentliche Voraussetzungen für eine leistungsbezogene Erfassung von Wirtschaftsfördermaßnahmen und deren Optimierung geschaffen werden.

\section{Die ABC-Analyse als Modell leistungs- bezogener Maßnahmenanpassung im Wirtschaftsförderbereich}

Angesichts der knapper werdenden Haushaltsmittel sind Entscheidungshilfen zur effizienten Mittelzuordnung auf die einzelnen Wirtschaftsförderinstrumente gefordert. In der unternehmerischen Managementpraxis hat sich als Planungsmodell zur kostenorientierten Zusammenstellung eines Leistungs- oder Nachfragemix die ABC-Analyse etabliert. Durch sie kann in anschaulicher Weise die Bedeutung verschiedener Leistungs- oder Beschaffungsprodukte für den unternehmerischen Erfolg erfasst werden. Gleichzeitig wird damit eine Grundlage für die Optimierung des Leistungs- oder Beschaffungsportfolios gelegt [7].

Mit dem Grundmodell der ABC-Analyse lassen sich auch die Auswirkungen der Ressourcenzuordnung auf die einzelnen Wirtschaftsförderfelder in Bezug auf deren Wirtschaftsfördererfolg darstellen und in Grenzen optimieren. Zur Veranschaulichung dient Abb. 2.

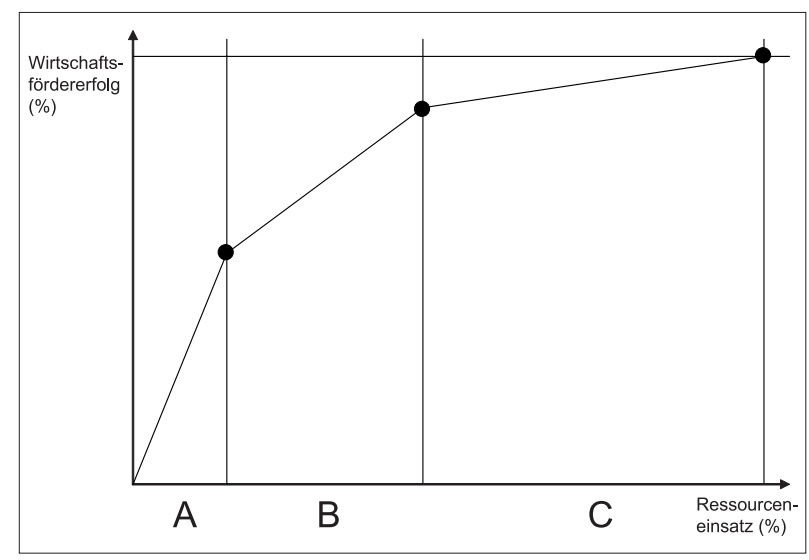

Abb. 2: ABC-Modell zur optimierten Wirtschaftsförderung

Auf der horizontalen Achse sind die Ressourceneinsätze (z. B. um eingeworbene Drittmittel reduzierte Finanzbudgets) für die einzelnen Wirtschaftsförderinstrumente (A, B, C) normiert auf $100 \%$ eingetragen. Auf der senkrechten Achse werden die den einzelnen Wirtschaftsförderinstrumenten zugeordneten Erfolgsgrößen ebenfalls auf $100 \%$ normiert erfasst. Die Reihenfolge des Eintrages der Wirtschaftsförderinstrumente auf der horizontalen Achse richtet sich nach dem abnehmenden Beitrag zum Wirtschaftsfördererfolg.

Das Verhältnis von instrumentenbezogenem Wirtschaftsfördererfolg (Nutzen) zu den dafür notwendigen Ausgaben (um eingeworbene Drittmittel reduziertes Finanzbudget) kann man als Grenznutzen oder Wirtschaftsförderproduktivität bezeichnen. In unserem Beispiel ist die Wirtschaftsförderproduktivität des Instrumentes A größer als B, diese wiederum größer als C.

Anhand des Modells lassen sich nunmehr verschiedene Budgetszenarien durchspielen: Bei einer Verschiebung der Budgetanteile, z. B. zu Lasten von A und zu Gunsten von $C$, würde der Gesamtwirtschaftsfördererfolg (ceteris paribus) sinken. Der Grund dafür ist, dass Budgetanteile von einem Instrument höherer Wirtschaftsförderproduktivität zu einem Instrument geringerer Wirtschaftsförderproduktivität verschoben wurden. Auch der Fall einer Verringerung des Gesamtbudgets lässt sich modellmäßig durchspielen. Die Reduzierung des Wirtschaftsfördererfolges kann dann in Grenzen gehalten werden, wenn zunächst die Maßnahmen mit der geringsten Wirtschaftsförderproduktivität eingeschränkt werden.

Die modellmäßige Betrachtung macht deutlich, dass eine erfolgsbezogene Optimierung des Instrumentenmix im Wirtschaftsförderbereich stets auch die Berïcksichtigung des jeweils notwendigen Budgeteinsatzes erfordert. Erst eine Betrachtung der Wirtschaftsförderproduktivitäten macht eine strukturierte Analyse möglich.

\section{Notwendigkeit und Grenzen praktischer Anwendung}

Enger werdende Haushaltsbudgets verlangen auch eindeutigere Prioritätensetzung bei der Wirtschaftsförderung. Die vorangegangenen Überlegungen zeigen, wie man auch bei knapper werdenden Budgets den Rückgang des Wirtschaftsfördererfolges in Grenzen halten kann. Durch die Orientierung an Produktivitätsüberlegungen der einzelnen Wirtschaftsförderinstrumente lässt sich die Effizienz des öffentlichen Mitteleinsatzes steigern. Hierzu kann das Instrument der ABC-Analyse geeignete Anhaltspunkte liefern. Es leistet insoweit auch einen Beitrag zur notwendigen Budgetoptimierung.

In seiner praktischen Anwendung erfordert das Modell zunächst einmal die getrennte Erfassung der Kosten/ Ausgaben für die einzelnen Instrumentenbündel der Wirtschaftsförderung, etwa für die Bereiche Existenzgründung, Unternehmensentwicklung und Ansiedlung. Sodann sind die Zielbeiträge je Instrumentenbïndel zu ermitteln, um die Produktivitäten der Instrumente berechnen und sie anschließend in eine Rangfolge bringen zu können. Die Maßstäbe der Messung des Wirtschaftsförder-Outputs können dabei sehr unterschiedlich sein. Auf die Problematik der eindimensionalen Orientierung an geschaffenen oder erhaltenen Arbeitsplätzen wurde hingewiesen und Erweiterungsmöglichkeiten entwickelt.

Allerdings findet der budgetbezogene Optimierungsprozess dort seine Grenzen, wo durch wechselseitige Abhängigkeiten zwischen den verschiedenen Instrumentenbündeln Einflüsse auf deren Wirksamkeit auftreten. Beispiele könnten sein, wenn durch verringerte Bestandspflege die Attraktivität eines Standortes für Ansiedlungen nachlässt oder durch übermäßige Vernachlässigung von 
Ansiedlungen Möglichkeiten des Aufbaus regionaler Zulieferketten zur Unternehmensentwicklung verloren gehen. Daher sind neben einer rein quantitativen Modellbetrachtung qualitative Gesichtspunkte möglicher Interdependenzen zwischen den Maßnahmenbündeln zu berüicksichtigen.

Empirische Erhebungen zur Anpassung von Wirtschaftsförderaktivitäten sind selten. Einen Anhaltspunkt liefert die regelmäßig von der Firma ExperConsult durchgefuihrte Befragung zum Thema „Wo steht die Wirtschaftsförderung in Deutschland“ - zuletzt für das Jahr 2002 [8]. Dort ist u. a. erkennbar, dass gemessen an den geschaffenen Arbeitsplätzen in 2002 die Gewichtung des Aufgabenschwerpunktes Bestandspflege mit durchschnittlich $64 \%$ (1999: $58 \%$ ) gegenüber dem Aufgabenschwerpunkt Neuansiedlung mit durchschnittlich $36 \%$ (1999: $42 \%$ ) deutlich zugenommen hat [9]. Dabei gewinnt das Instrument Netzwerk- und Clusterbildung zunehmende Bedeutung.

Auch bei der staatlichen Wirtschaftsförderung kann es kein „weiter wie bisher“ geben. Dazu haben sich die Rahmenbedingungen zu sehr verändert - sowohl bei den strukturellen Anforderungen als auch bei den haushaltswirtschaftlichen Möglichkeiten. Mehr denn je sind Effizienzbetrachtungen bei den Wirtschaftsförderinstrumenten notwendig, mit denen Kosten und Nutzen einander gegenuiber gestellt werden können. Dieser Beitrag soll die Diskussion anregen und mögliche Denkrichtungen aufzeigen.

\section{Anmerkungen/Literatur}

[1] Schulze, Klaus-P.: Wirtschaft im Wandel - Anforderungen an eine zukunftsorientierte Wirtschaftsförderung, in: TFH Wildau (Hrsg.); Wissenschaftliche Beiträge, Heft 2002, S. $71 \mathrm{ff}$.

[2] McKinsey \& Company: Deutschland übermorgen - Verstärkung der regionalen wirtschaftlichen Leistungsfähigkeit und Schaffung neuer Arbeitsplätze, Vortragsunterlagen

[3] Bundesministerium für Bildung und Forschung, BMBF (Hrsg.): Zur technologischen Leistungsfähigkeit Deutschlands 2002, Bonn 2003

[4] BMBF (Hrsg.): a.a.O., S. 5

[5] BMBF (Hrsg.): a.a.O., S. $173 \mathrm{ff}$.

[6] Steden, P. in Prognos (Hrsg.): trendletter 1/2003, S. 10

[7] Heinen, Edmund: Industriebetriebslehre - Entscheidungen im Industriebetrieb, 9. Aufl., Wiesbaden 1991, S. 500 ff.

[8] ExperConsult, Wirtschaftsförderung und Investitionen GmbH \& Co. KG, „Wo steht die Wirtschaftsförderung in Deutschland? - Befragung 2002“, Dortmund 2002

[9] ExperConsult, a.a.O., S. 14

\section{Autor}

Prof. Dr. rer. pol. Klaus-Peter Schulze

Geschäftsfuihrer

ZukunftsAgentur Brandenburg GmbH

Steinstraße 104-106, 14480 Potsdam

Tel. +49331660-3833

E-Mail: klaus-peter.schulze@zab-brandenburg.de 